\title{
Broadband Back Grating Design for Thin Film Solar Cells
}

\author{
Bilal Janjua, Ghassan Jabbour* \\ Solar Center, Photonics Laboratory, Computer, Electrical, and Mathematical Sciences and Engineering (CEMSE) Division, King \\ Abdullah University of Science \& Technology (KAUST), Thuwal 23955-6900, Kingdom of Saudi Arabia (KSA)
}

\begin{abstract}
In this paper, design based on tapered circular grating structure was studied, to provide broadband enhancement in thin film amorphous silicon solar cells. In comparison to planar structure an absorption enhancement of $\sim 7 \%$ was realized.
\end{abstract}

Thin film solar cells based on amorphous silicon (a-Si), have the potential to replace $\mathrm{c}-\mathrm{Si}$ by providing a cheap alternative.

Limitations in current texturing designs require the need to explorer new physical phenomena's to enhance light trapping in thin film solar cells. Surface Plasmon (SP) on metallic nanostructures (NS) is one such field, which has shown to boost absorption in a-Si thin film solar cells [1]. SP is the collective oscillation of electrons in response to an applied electric field. The motion of the electron causes light scattering, near field enhancement (localized Surface Plasmon (LSP)) and surface propagating waves (Surface Plasmon Polariton (SPP)) at metal dielectric interface.

Current designs, based on metallic NPs placed on the front contact, provide an easy means of incorporating plasmonic effects in solar cell. NPs can be designed to scatter long wavelength radiation thus increasing the optical path in the absorbing region. The drawback of placing NP on the top contact is, scattering of short wavelength radiation, due to fanointerference effects [2]. Shadowing effects further limits the percentage coverage of NP thus reducing device performance.

An intelligent way to mitigate reflection and interference related loses is to incorporate plasmonic NS at back contact [3, $4,5]$. Conformal texturing of the top surface produces graded refractive index resulting in reduced reflection losses and improved device performance.

In this paper, a novel back grating design based on tapered circular holes, as back reflector, is proposed. This design can provide a broadband and polarization insensitive absorption enhancement by scattering and coupling of incident light to waveguide (WG), LSP and SPP modes. Rigorously electromagnetic simulations were done using commercially available finite element software (Comsol 4.3). Tapered circular structures can be fabricated using electron beam lithography (EBL) followed by a wet etching process of [100] silicon facet. To expedite the EBL process, nano-imprint lithography (NIL) can be adopted.

The structure of the device being simulated consists of a silver (Ag) tapered circular grating at back contact. On top of the grating, a thin zinc oxide ( $\mathrm{ZnO})$ layer is used to suppress SPP related losses in metal [6]. Indium tin oxide (ITO) is used as a transparent conducting oxide (TCO) for top contact. Amorphous silicon (a-Si) acting as the absorbing medium is sandwiched between the ITO and $\mathrm{ZnO}$ layers. Thickness of a-
$\mathrm{Si}, \mathrm{ZnO}$ and ITO are fixed at 200, 130 and $80 \mathrm{~nm}$, for simplicity. The grating slits are filled with $\mathrm{ZnO}$. Conformal texturing of top layers is neglected in this study. TE light was incident from the top, via a port.

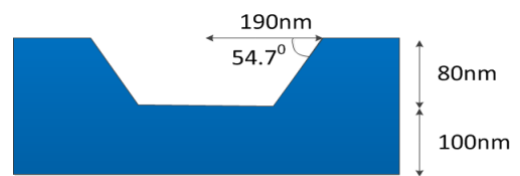

Fig. 1. Shown is the cross sectional view of the proposed grating design unit cell.

Rigorous simulations were done to optimize the height of the grating and the opening radius of the slit. As shown in fig.1, best results were achieved for slit radius of $190 \mathrm{~nm}$ with grating height of $80 \mathrm{~nm}$ and period $450 \mathrm{~nm}$. The grating was placed on $100 \mathrm{~nm}$ Ag back contact. This ensured negligible transmission losses. The tapered edges, made an angle of $54.7^{\circ}$ with the horizontal plane. This depicts a thin $\mathrm{Ag}$ coating on an anisotropically wet etched silicon wafer.

Taking into account the solar spectrum, a wavelength range of 300 to $1200 \mathrm{~nm}$ was selected for simulation. Dispersive dielectric constant used for materials were taken from reference [7] to [9]. The computational domain consisted of a unit cell surrounded by a perfect electric boundary condition (PEC) and perfect magnetic boundary conditions (PMC). Absorption in the layers was calculated using:

$$
\text { Absorption }=\oint s \vec{S}(\vec{r}, w) \cdot d \vec{a}
$$

where $\vec{S},(\vec{r}, w)$ the pointing vector and $\mathrm{s}$ is the surface of the layer being analyzed.

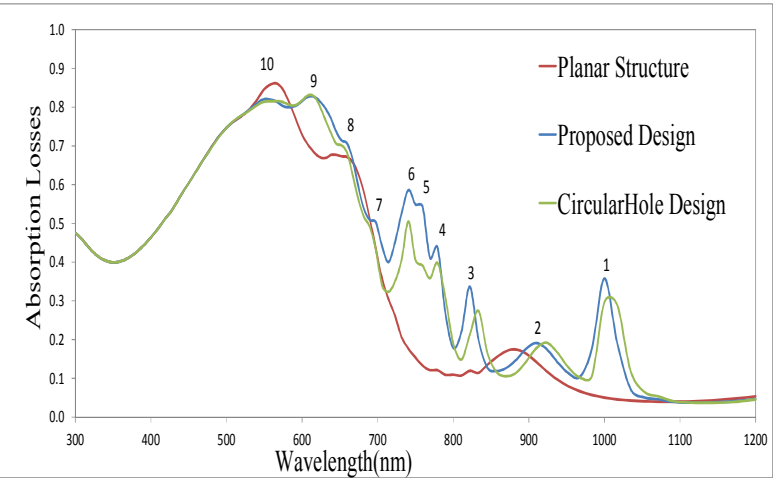

Fig. 2. Absorption losses in a-Si layer, for different structures are plotted Peaks 1-10, refer to excitation of different localized modes in the proposed design. For comparison absorption losses in reference planar and circular hole based structure is also plotted. 
Absorption loss in a-Si layer was plotted in fig. 2. For comparison, results of planar and circular hole design, without tapered edges, are also plotted. An enhancement of $\sim 7 \%$ was realized in absorption for the proposed designed, in comparison to planar structure.

As can be seen on fig. 2, at long wavelengths, peaks 1-10, appear in the proposed design. The peaks correspond to coupling of incident light into different EM localized modes supported by the structure.

In fig. 2, peaks 2, 8 and 10 are due to cavity modes or Fabry Perot (FP) resonances in a-Si layers. Cavity resonances occur due to inference effects of EM waves. Similar peaks can also be seen on the absorption plot for reference planar structure. Peak 2 in fig. 2, shows a red shift for proposed design compared to planar structure. The shift is due to change in effective cavity thickness, in presence of the metallic silver grating and $\mathrm{ZnO}$ region in the grating slits [3].

As can be seen in fig. 2, apart from peaks due to FP resonances, additional peaks appear in the absorption plot for the proposed design. The new peaks are assigned to excitation of waveguide modes supported by the structure. Cross sectional view of the EM fields, not shown in this paper, support the analysis made on fig. 2 .

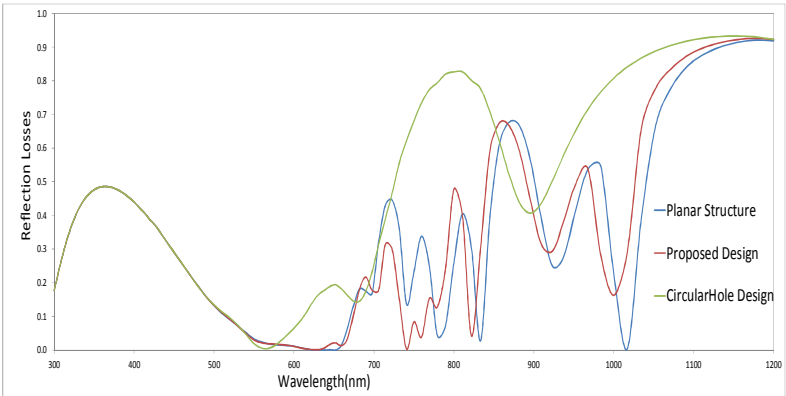

Fig. 3, Reflection losses are plotted for structures being studied. Reflection losses are calculated using S11 parameter.

In fig. 3, reflection losses at different wavelengths, was plotted for reference planar structure, proposed and circular hole design, without tapered edges. Reflection losses were measured using the S11 scattering parameter. Absorption enhancement, in the range of 650 and $800 \mathrm{~nm}$, for the proposed design, compared to structure with non-tapered holes was attributed to reduced reflection losses. For planar structure, high reflection losses at long wavelengths are due to lack of coupling of incident light into localized modes of the structure.

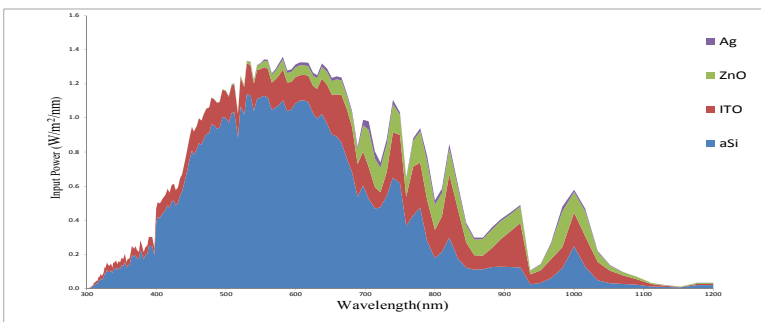

Fig. 4, Absorption Losses in different layer of the proposed design is plotted using stacked area chart. Calculated absorption for unit incident power is normalized with AM 1.5 solar spectrum.
In fig. 4, a stacked area chart was plotted to compare absorption losses in different layers of the proposed structure. Absorption losses with unit incident power were weighted by air mass (AM) 1.5 solar spectrum for wavelengths 250 to $1200 \mathrm{~nm}$. As shown in fig. 4, major part of absorption takes place in the a-Si layer which contributes to useful solar current. For wavelengths greater than $600 \mathrm{~nm}$, losses in ITO and $\mathrm{ZnO}$ become relevant with little contribution from the metal. This is due to low absorption coefficient of a-Si layer near its band gap energy. As shown in fig. 3, reflection losses also increase at longer wavelengths. Relatively small losses in the metal are due to suppression of SPP modes via use of thin $\mathrm{ZnO}$ layer.

By choosing a circular design with tapered edges, a broad band scattering response of the dipoles is expected. A tapered design provides a linearly graded phase, imparted to the incident wave. This reduces the escaping of light via specular reflection of zero order modes, over a wide range of wavelengths. Thus enhancement in light absorption is realized by using a novel plasmonic grating design for back reflection in thin film solar cells. Such sub wavelength periodic structures not only diffracts and scatter incident light but also couple radiation into supported localized modes. For further optimization of the proposed design, absorption losses in the TCO layers can become the limiting factor. Incorporating texturing in subsequent layer, should realize a more realistic model. Conformal texturing, in theory, will further reduce reflection losses at ITO/air interface and enhance device performance.

In this paper, light trapping in a-Si layer using a novel grating design was studied. Simulations were done on commercially available FEM software (Comsol v.4.3). The proposed design consisted of a grating structure based on tapered circular holes. The enhancement in absorption was attributed to coupling of light into WG, FP and SPP modes. For longer wavelengths, absorption in the TCO layers limited device performance. Compared to reference planar structure, absorption enhancement of $\sim 7 \%$ was realized for the proposed grating design. Device response to change of angle of incidence of input light will be a part of future study.

\section{REFERENCES}

[1] W. L Barnes, A. Dereux, T. W. Ebbesen, "Surface plasmon subwavelength optics," Nature, vol. 424, p. 824-830, 2003.

[2] F. J. Beck, A. Polman, and K. R. Catchpole, "Tunable light trapping for solar cells using localized surface plasmons," J. Appl. Phys., vol. 105, no. 11, p. 114310, 2009

[3] W. Wang, S. Wu, et al, "Broadband light absorption enhancement in thinfilm silicon solar cells," Nano Lett., vol. 10, no. 6, 2010.

[4] Vivian E. Ferry, Marc A. Verschuuren, et al, "Improved red-response in thin film a-Si:H solar cells with soft-imprinted plasmonic back reflectors," Appl. Phys. Lett., vol. 95, p. 183503, 2009.

[5] R. H. Franken, R. L. Stolk, H. Li, C. H. M. van der Werf, J. K. Rath, and R. E. I. Schropp, "Understanding light trapping by light scattering textured back electrodes in thin film n-i-p-type silicon solar cells," J. Appl. Phys., vol. 102, no. 1, p. 014503, 2007.

[6] F. J. Haug, T. Sooderstroom, et al, "Plasmonic absorption in textured silver back reflectors of thin film solar cells," J. Appl. Phys., vol. 104, no. 6, p. 064509, 2008.

[7] P. B. Johnson, R. W. Christy, "Optical Constants of the nobel Metals," Phys Rev. B., vol. 6, no 12, 1972.

[8] Hisashi Yoshikawa, and Sadao Adachi, "Optical Constants of ZnO,” Jpn. J. Appl. Phys., vol. 36, p. 62367-6243, 1997.

[9] SOPRA n\&k Optical Database.

[10] K. R. Catchpole, A. Polman, "Plasmonic solar cells," Opt. Express vol. 16, p. 21793-21800, 2008. 